\title{
Pose-Invariant 3D Proximal Femur Estimation through Bi-planar Image Segmentation with Hierarchical Higher-Order Graph-Based Priors
}

\author{
Chaohui Wang ${ }^{1,2}$, Haithem Boussaid ${ }^{1,2}$, Loic Simon ${ }^{1}$, \\ Jean-Yves Lazennec ${ }^{3}$, and Nikos Paragios ${ }^{1,2, \star}$ \\ 1 Laboratoire MAS, Ecole Centrale de Paris, France \\ ${ }^{2}$ Equipe GALEN, INRIA Saclay - Île de France, Orsay, France \\ ${ }^{3}$ Centre Hospitalier Universitaire Pitié Salpêtrière, Paris, France
}

\begin{abstract}
Low-dose CT-like imaging systems offer numerous perspectives in terms of clinical application, in particular for osteoarticular diseases. In this paper, we address the challenging problem of 3D femur modeling and estimation from bi-planar views. Our contributions are threefold. First, we propose a non-uniform hierarchical decomposition of the shape prior of increasing clinical-relevant precision which is achieved through curvature driven unsupervised clustering acting on the geodesic distances between vertices. Second, we introduce a graphical-model representation of the femur which can be learned from a small number of training examples and involves third-order and fourth-order priors, while being similarity and mirror-symmetry invariant and providing means of measuring regional and boundary supports in the bi-planar views. Last but not least, we adopt an efficient dual-decomposition optimization approach for efficient inference of the 3D femur configuration from bi-planar views. Promising results demonstrate the potential of our method.
\end{abstract}

\section{Introduction}

Low-dose X-ray imaging has gained significant attention during the recent years, due to the increase of image quality and the decrease of the radiation exposure. Even though such images do not scale to computer tomography in terms of precision and resolution, they can be a valuable diagnostic tool for a number of diseases and in particular for osteopathies [10] like those related to spine [5], femur 9], etc. In this paper, we are interested in 3D proximal femur reconstruction focusing on highly accurate 3D modeling, in particular, for the femoral head part. In fact, patient specific 3D planning of the femoral head, inherits important diagnostic interest in related surgical interventions such as total hip replacement and intertrochanteric osteotomy [3].

Bone extraction and segmentation from bi-planar X-ray images is a challenging task due to the poor image quality, the fact that one has to compensate

\footnotetext{
* This work is supported by the European Research Council Starting Grant DIOCLES (ERC-STG-259112) and the MEDICEN Competitive Cluster sterEOS+ grant.
} 
the partial support (because of the 3D-to-2D projection), etc. In such a context, conventional stereo-vision techniques [5] that recover $3 \mathrm{D}$ measurements from $2 \mathrm{D}$ projections do fail to provide clinically interesting results either because of the lack of correct correspondences between the two views or due to the sparsity of the obtained measurements. Thus, prior knowledge is usually considered, where statistical models of proximal femur is learned from a set of training examples 4]. Point-distribution models and in particular active shape models (ASMs) [1] have become a main stream and were also used for femur segmentation [4. Pre-processing such as segmentation of femur contours [8] can facilitate the 3D femur pose estimation since the task is transformed into searching for a geometric mapping between the 3D model and the corresponding $2 \mathrm{D}$ multi-view silhouettes. Other methods act directly on the projection space and try to optimize global and local pose by measuring a projection energy, which can depend on key points 10, contours 9] or regional statistics [11. However, these methods inherit various limitations. They need registration for all training examples to a common pose and subsequently for the testing image. Furthermore, important training set has to be considered towards capturing the variability of the femur, due to the global representation of shape models. Last, the inference can also be quite problematic due to the use of gradient-driven methods or the need of pre-segmentation, which is very challenging because of low signal-to-noise ratio.

In this paper, we propose a novel approach for femur estimation. Our contributions are threefold. First, we propose a non-uniform hierarchical decomposition of the shape prior of increasing clinical-relevant precision which is achieved through curvature driven unsupervised clustering acting on the geodesic distances between vertices. Second, we introduce a higher-order graphical-model representation of the femur which can be learned from a small number of training examples and involves third-order and fourth-order priors, while being similarity and mirror-symmetry invariant and providing means of measuring regional and boundary supports in the bi-planar views. Last but not least, we adopt an efficient dual-decomposition optimization approach for efficient inference of the 3D femur configuration from bi-planar views, leading to promising results.

The reminder of the paper is organized as follows: we present our hierarchical shape representation with the corresponding prior in Sec. 2, and a decomposed observation model in Sec. 3. They are combined within a probabilistic formulation which is then transformed into a higher-order MRF towards femur estimation in Sec. 4, Experimental results compose Sec. 5, while discussion and future work conclude the paper in Sec. 6.

\section{Hierarchical Multi-resolution Probabilistic Modeling}

In the literature, a surface is usually modeled as a mesh with uniform resolution. However, some anatomical regions (e.g., femoral head) are of higher clinical relevance than adjacent parts. Hence, we propose to construct a hierarchical multi-resolution representation (Fig. 1(a)) of the femur with different clinicalrelevant precisions, so that computational effort can be focused on the parts that are meant to be reconstructed with high fidelity. 


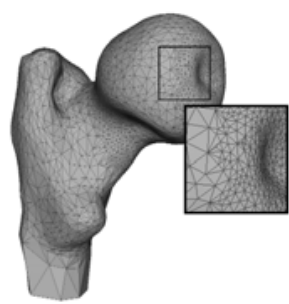

(a)

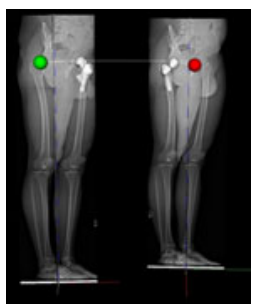

(b)

Fig. 1. (a) Multi-resolution surface Model. (b) EOS System.

First, mesh sub-sampling from one level $\mathcal{V}_{m}$ to a coarser one $\mathcal{V}_{m+1} \subset$ $\mathcal{V}_{m}$ is performed iteratively, starting from a given high-resolution mesh $\mathcal{M}_{0}=$ $\left(\mathcal{V}_{0}, \mathcal{E}_{0}, \mathcal{F}_{0}\right)(\mathcal{V}$ denotes vertex set, $\mathcal{E}$ edge set and $\mathcal{F}$ face set), to create a nonincreasing sequence of vertex subsets $\mathcal{V}_{0} \supset \cdots \supset \mathcal{V}_{M}$, where each subset stands for a level of detail in the multi-resolution hierarchy. We propose to formulate such a sub-sampling problem as a clustering (Eq. 1) based on geodesic distances and curvatures, aiming to obtain a subset $\mathcal{V}_{m+1}$ of points uniformly distributed on the surface while preserving preferentially regions of high curvature:

$$
\mathcal{V}_{m+1}=\underset{\mathcal{V} \subset \mathcal{V}_{m}}{\operatorname{argmin}}\left[\sum_{v \in \mathcal{V}_{m}} \min _{\hat{v} \in \mathcal{V}} d(v, \hat{v})+\alpha \sum_{\hat{v} \in \mathcal{V}} \exp (-\operatorname{curv}(\hat{v}))\right]
$$

where $d(v, \hat{v})$ is the geodesic distance between $v$ and $\hat{v}$ on $\mathcal{M}_{0}, \alpha$ is a positive weight and $\operatorname{curv}(\hat{v})$ is the curvature at $\hat{v}$ on $\mathcal{M}_{0}$. The higher $\operatorname{curv}(\hat{v})$ is, the easier $\hat{v}$ will be promoted as cluster center. Hence, this formulation tends to promote more cluster centers in the regions of high curvature. It can be efficiently solved through linear programming techniques proposed in [7] and the obtained $\mathcal{V}_{m+1}$ corresponds to the cluster centers and each $v \in \mathcal{V}_{m}$ is associated to the closest center. One main particularity of this approach is that, as opposed to classical approaches (e.g., K-means), the number of clusters is controlled by the penalty $\exp (-\operatorname{curv}(\hat{v}))$ which is related to flatness of the surface.

Second, level of detail selection is performed to select vertices from the sets $\left(\mathcal{V}_{m}\right)_{m=0, \ldots, M}$ so that different regions are represented at given resolutions. We organize the vertices in a tree structure, since any vertex in $\mathcal{V}_{m}$ is associated to one and only one vertex in $\mathcal{V}_{m+1}$ in the hierarchical structure derived from the previous step. Then starting from the coarsest resolution, one can select the regions to be refined and iterate this process until reaching the required accuracy for every part. Through this step we obtain a set of vertices $\mathcal{V}_{\mathrm{MR}}$.

Last, connectivity computation is performed on $\mathcal{V}_{\mathrm{MR}}$ in order to achieve the triangulated multi-resolution mesh. To this end, we compute the Delaunay triangulation of $\mathcal{V}_{\mathrm{MR}}$ associated to the geodesic distance. By viewing the Delaunay triangulation as the dual of the Voronoi diagram of $\mathcal{V}_{\mathrm{MR}}$, it boils down to determining which pairs of Voronoi cells have a common boundary. In this way, 
we obtain a set of edges $\mathcal{E}_{\mathrm{MR}}$. Then the corresponding faces $\mathcal{F}_{\mathrm{MR}}$ are computed to achieve the mesh, by searching for minimal cycles in the edge list.

The proposed approach provides an economic way to model the surface according to the clinical relevance and a significantly better approximation of the original mesh compared with the classic edge collapse techniques. Furthermore, the vertices of the obtained mesh are among those of the original mesh, which facilitates largely the model prior learning. Finally, let $(\mathcal{V}, \mathcal{E}, \mathcal{F})$ denote the vertex set $\mathcal{V}_{\mathrm{MR}}$, the edge set $\mathcal{E}_{\mathrm{MR}}$ and the face set $\mathcal{F}_{\mathrm{MR}}$ of the obtained mesh. Then the surface is parametrized by the $3 \mathrm{D}$ positions $\mathbf{u}=\left(u_{i}\right)_{i \in \mathcal{V}}$ of all the vertices.

Probabilistic Shape Modeling: The training data to learn statistical model consist of the 3D positions of the vertices on the training surfaces. No assumption on registration between surfaces is being made, we only assume that correspondences have been determined for the vertices among the training samples.

We adopt the pose-invariant prior in [12 and extend it in a more general formulation. Such a prior does not require the estimation of global pose in the training and testing stages, which eliminates the bias caused by such estimations. Furthermore, this prior is mirror-symmetry-invariant, leading to one more advantage in our problem: a common statistical model can be used for both the left and right femurs. For a clique $c(c \subseteq \mathbf{V}$ and $|c| \geq 3)$ of vertices, we enumerate all the pairs $\mathcal{P}_{c}=\{(i, j) \mid i, j \in c$ and $i<j\}$ of points and compute the relative distance $\hat{d}_{i j}=d_{i j} / \sum_{(i, j) \in \mathcal{P}_{\mathbf{c}}} d_{i j}$ for a pair of points $(i, j) \in \mathcal{P}_{c}$, where $d_{i j}=\left\|u_{i}-u_{j}\right\|$ denotes the Euclidean distance between points $i$ and $j$. Since for clique $c$, any relative distance $\hat{d}_{i j}$ is a linear combination of the others (i.e., $\left.\sum_{(i, j) \in \mathcal{P}_{c}} \hat{d}_{i j}=1\right)$, we put all the relative distances except one (denoted as $\bar{p}$ ) in a vector $\hat{\mathbf{d}}_{c}=\left(\hat{d}_{i j}\right)_{(i, j) \in \mathcal{P}_{c} \backslash\{\bar{p}\}}$. We model the distribution $\psi_{c}\left(\hat{\mathbf{d}}_{c}\right)$ of $\hat{\mathbf{d}}_{c}$ using Gaussian Mixture Models (GMMs) which are learned from the training data.

In order to enforce the smoothness of the surface, for each quadruplet $q$ of vertices corresponding to a pair of adjacent facets, we introduce a smoothness potential function encoding constraints on the change of the normal directions:

$$
\psi_{q}\left(\mathbf{u}_{q}\right)=\exp \left\{-\left(1-<\vec{n}_{q}^{(1)}\left(\mathbf{u}_{q}\right), \vec{n}_{q}^{(2)}\left(\mathbf{u}_{q}\right)>\right) / \beta\right\}
$$

where $\mathbf{u}_{q}$ denotes the $3 \mathrm{D}$ positions of the four vertices contained in $q,\langle\cdot, \cdot\rangle$ denotes the scalar product, $\beta$ is a positive constant, and $\vec{n}_{q}^{(1)}, \vec{n}_{q}^{(2)}$ denote the unit normal vectors of the two facets.

Finally, let $\mathcal{T}=\{c \mid c \subseteq \mathcal{V}$ and $|c|=3\}$ denotes the set of triplets1, and $\mathcal{Q} \subset\{q \mid q \subseteq \mathcal{V}$ and $|q|=4\}$ denote the set of quadruplets corresponding to all pairs of adjacent facets, then the prior probability of the $3 \mathrm{D}$ model is defined as:

$$
p(\mathbf{u}) \propto \prod_{c \in \mathcal{T}} \psi_{c}\left(\hat{\mathbf{d}}_{c}\left(\mathbf{u}_{c}\right)\right) \cdot \prod_{q \in \mathcal{Q}} \psi_{q}\left(\mathbf{u}_{q}\right)
$$

\footnotetext{
${ }^{1}$ We use 3-order cliques for the corresponding prior term, i.e., $|c|=3$. However, other higher-order cliques $c(|c| \geq 3)$ can also be used.
} 


\section{Observation Model}

Let $\mathbf{I}=\left(I_{k}\right)_{k \in \mathcal{K}}$ (where $\mathcal{K}=\{1, \ldots, K\}, K=2$ for the case of bi-planar views) denote $K$ observed images captured from different viewpoints with the corresponding projection matrices $\boldsymbol{\Pi}=\left(\Pi_{k}\right)_{k \in \mathcal{K}}$. By exploiting the conditional independency, the image likelihood $p(\mathbf{I} \mid \mathbf{u}, \boldsymbol{\Pi})$ is decomposed into:

$$
p(\mathbf{I} \mid \mathbf{u}, \boldsymbol{\Pi})=\prod_{k \in \mathcal{K}} p\left(I_{k} \mid \mathbf{u}, \Pi_{k}\right)
$$

We introduce a decomposed formulation for the likelihood $p\left(I_{k} \mid \mathbf{u}, \Pi_{k}\right)$ of the observed image $k$, which can be easily encoded in a higher-order MRF towards efficient inference. $p\left(I_{k} \mid \mathbf{u}, \Pi_{k}\right)$ is modeled as a Gibbs distribution, combining both the region-based and boundary-based data supports:

$$
p\left(I_{k} \mid \mathbf{u}, \Pi_{k}\right) \propto \exp \left\{-\frac{\lambda E_{k}^{\mathrm{R}}\left(I_{k}, \mathbf{u}, \Pi_{k}\right)+(1-\lambda) E_{k}^{\mathrm{B}}\left(I_{k}, \mathbf{u}, \Pi_{k}\right)}{T_{k}}\right\}
$$

where $T_{k}$ is temperature, and $0<\lambda<1$ is a balancing weight coefficient.

Regional Term. $E_{k}^{\mathrm{R}}$ encodes the statistical intensity properties of the two classes (femur and non-femur) and guides the projections of the surface to match the silhouettes of the femur in the observed images. We define this term as a sum of likelihoods on the projections of the front-facing facets:

$$
E_{k}^{\mathrm{R}}\left(I_{k}, \mathbf{u}, \Pi_{k}\right)=\sum_{f \in \mathcal{F}} \delta_{f}\left(\mathbf{u}_{f}, \Pi_{k}\right) \cdot \iint_{\Omega\left(\mathbf{u}_{f}, \Pi_{k}\right)} \log \frac{p_{b g}(I(x, y))}{p_{f g}(I(x, y))} d x d y
$$

where $f$ denotes a triangular facet, $\mathbf{u}_{f}$ denotes the $3 \mathrm{D}$ coordinates of the three vertices of $f$, the indicator function $\delta_{f}\left(\mathbf{u}_{f}, \Pi_{k}\right)$ is equal to 1 if the facet $f$ is front-facing w.r.t. the camera and 0 otherwise, $\Omega_{f}\left(\mathbf{u}_{f}, \Pi_{k}\right)$ is the $2 \mathrm{D}$ region corresponding to the projection of $f, p_{f g}$ and $p_{b g}$ denote the distributions (modeled using GMMs) of the intensity for the regions of the femur and the background.

Boundary Term. $E_{k}^{\mathrm{B}}$ encodes discontinuities along the boundaries. Recall that $\mathcal{Q}$ denotes the set of quadruplets of vertices for the adjacent facets sharing an edge $e \in \mathcal{E}$. For each quadruplet $q \in \mathcal{Q}$, we define a function $\delta_{q}\left(\mathbf{u}_{q}, \Pi_{k}\right)$ which is equal to 1 (i.e., the projection of $e$ is a piece of boundary) when the facet closer to the camera is front-facing and the other one is back-facing, and 0 otherwise. We define the boundary term as a sum of the discontinuity measurement for all these quadruplets:

$$
E_{k}^{\mathrm{B}}\left(I_{k}, \mathbf{u}, \Pi_{k}\right)=\sum_{q \in \mathcal{Q}} \delta_{q}\left(\mathbf{u}_{q}, \Pi_{k}\right) \cdot \int_{\Gamma\left(\mathbf{u}_{q}, \Pi_{k}\right)}<\nabla I_{k}(x, y), \overrightarrow{n(x, y)}>d s
$$

where $\Gamma\left(\mathbf{u}_{q}, \Pi_{k}\right)$ denotes the projection of the edge shared by the two adjacent facets, $\overrightarrow{n(x, y)}$ denotes the outward-pointing unit normal of $\Gamma\left(\mathbf{u}_{q}, \Pi_{k}\right)$, $\nabla I_{k}(x, y)=\left(\frac{\partial I_{k}(x, y)}{\partial x}, \frac{\partial I_{k}(x, y)}{\partial y}\right)$ denotes the gradient of the intensity at $(x, y)$. Note that other boundary discontinuity measurements can also be used as the integrand in Eq. 7. 


\section{Probabilistic 3D Surface Estimation Framework}

The 3D model prior $p(\mathbf{u})$ (Sec. 2) and the image likelihood $p(\mathbf{I} \mid \mathbf{u}, \boldsymbol{\Pi})$ (Sec. 3) can be combined through a Bayesian framework, where the 3D surface estimation is formulated as a maximization of the posterior probability of $\mathbf{u}$ :

$$
\mathbf{u}^{\text {opt }}=\underset{\mathbf{u} \in \mathcal{U}}{\operatorname{argmax}} p(\mathbf{u} \mid \mathbf{I}, \boldsymbol{\Pi})
$$

And the posterior probability $p(\mathbf{u} \mid \mathbf{I}, \boldsymbol{\Pi})$ is:

$$
p(\mathbf{u} \mid \mathbf{I}, \boldsymbol{\Pi})=\frac{p(\mathbf{u}, \mathbf{I}, \boldsymbol{\Pi})}{p(\mathbf{I}, \boldsymbol{\Pi})} \propto p(\mathbf{u}, \mathbf{I}, \boldsymbol{\Pi})=p(\mathbf{I} \mid \mathbf{u}, \boldsymbol{\Pi}) p(\mathbf{u}) p(\boldsymbol{\Pi}) \propto p(\mathbf{I} \mid \mathbf{u}, \boldsymbol{\Pi}) p(\mathbf{u})
$$

Higher-order MRF Formulation: The probabilistic framework above can be easily reformulated within a higher-order MRF so that we can employ efficient MRF inference algorithms to achieve femur reconstruction. To this end, we use a node to model a vertex $i(i \in \mathcal{V})$ with its latent 3D position $U_{i}$, a third-order clique $c(c \in \mathcal{T})$ to model a triplet of vertices, and a fourth-order clique $q(q \in \mathcal{Q})$ to model the quadruplets of vertices corresponding to the adjacent facets. The $3 \mathrm{D}$ model is estimated through minimizing the MRF energy $E(\mathbf{u})$ :

$$
\mathbf{u}^{\mathrm{opt}}=\underset{\mathbf{u} \in \mathcal{U}}{\operatorname{argmin}} E(\mathbf{u})
$$

where $E(\mathbf{u})=-\log p(\mathbf{u} \mid \mathbf{I}, \boldsymbol{\Pi})+$ constant, and can be factorized into:

$$
E(\mathbf{u})=\sum_{f \in \mathcal{F}} H_{f}^{R}\left(\mathbf{u}_{f}\right)+\sum_{q \in \mathcal{Q}}\left(H_{q}^{B}\left(\mathbf{u}_{q}\right)+H_{q}^{P}\left(\mathbf{u}_{q}\right)\right)+\sum_{c \in \mathcal{T}} H_{c}^{P}\left(\mathbf{u}_{c}\right)
$$

Regional-term potentials $H_{f}^{R}\left(\mathbf{u}_{f}\right)(f \in \mathcal{F})$ encode the regional data term $E_{k}^{\mathrm{R}}\left(I_{k}, \mathbf{u}, \Pi_{k}\right)$ (Eq. 6). Boundary-term potentials $H_{q}^{B}\left(\mathbf{u}_{q}\right)(q \in \mathcal{Q})$ encode the boundary term $E_{k}^{\mathrm{B}}\left(I_{k}, \mathbf{u}, \Pi_{k}\right)$ (Eq. 7). Model Prior Potentials $H_{c}^{P}\left(\mathbf{u}_{c}\right)(c \in \mathcal{T})$ and $H_{q}^{P}\left(\mathbf{u}_{q}\right)(q \in \mathcal{Q})$ encode the prior on the relative positions of points and the prior on the smoothness, respectively (Eq. 3). Through the negative logarithmic operation, we can obtain their definitions as follows:

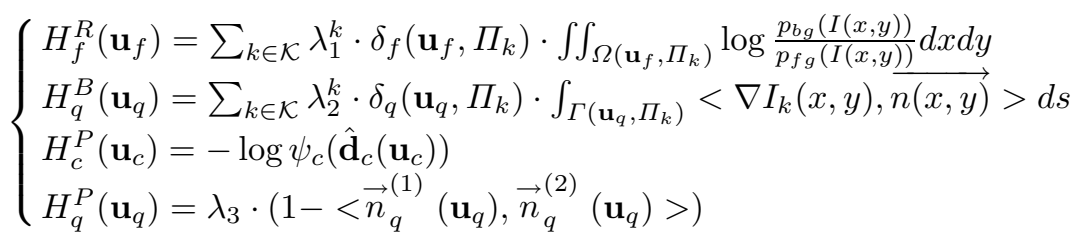

where $\lambda_{1}^{k}, \lambda_{2}^{k}$ and $\lambda_{3}$ are positive constant weights 2 for the corresponding terms.

Dual-Decomposition MRF Inference. [6] was adopted to optimize the proposed higher-order MRF. Like [12, we decompose the original graph into a set of factor trees which can be solved within polynomial time using max-product belief propagation. Their solutions are combined using projected subgradient method [6] to achieve the solution of the original problem.

\footnotetext{
${ }^{2}$ We use a single constant $\lambda_{1}$ for $\lambda_{1}^{k}(\forall k)$, and a single constant $\lambda_{2}$ for $\lambda_{2}^{k}(\forall k)$.
} 


\section{Experimental Validation}

We use the low-dose EOS imaging system (BiospaceMed ${ }^{\mathrm{TM}}$ ) which is an alternative system that captures simultaneously bi-planar X-ray images, coronal and sagittal in an upright position (Fig. 1(b)). The training set consists of 20 CT patient volumes of the hip. Manual segmentations of them are provided to extract femoral surfaces, which are used to learn the model prior. With the obtained prior, we perform the femur reconstruction using the proposed method. The search of the model parameters is done using the coarse-to-fine scheme and the sparse sampling strategy as in [2].

The proposed method has been validated using both dry femurs and real clinical data. The in vitro testing data consist of 14 pairs of EOS images, for which the ground truth surfaces are provided by the gold standard CT method. The bones are of real size for adults. Quantitative evaluation was conducted by comparing the estimated surfaces to the ground truths, with respect to the DICE coefficient and the distances from the 3D vertices of the estimated femur to the ground truth surface (called point-to-surface error). Fig. 2(a) shows four samples from the set of results, with point-to-surface error on femoral head. Fig. 2(b) presents the statistics of the DICE coefficients, the mean and standard deviation of the point-to-surface errors for the whole in vitro validation set. Four in vivo examples were also tested, where both left and right femurs were constructed from the EOS images. The qualitative results in Fig. 2( $(\mathrm{c}-\mathrm{d})$ show that the projections of the reconstructed surfaces fit well the femurs in the observed images despite complex scene and low image quality.

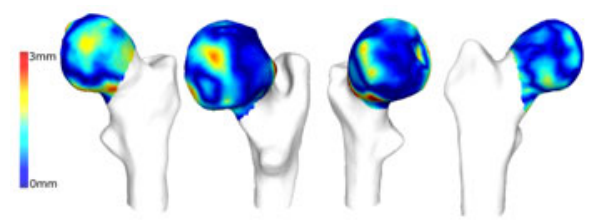

(a)
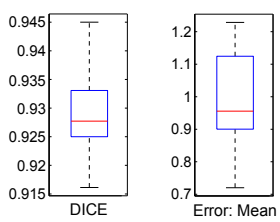

(b)

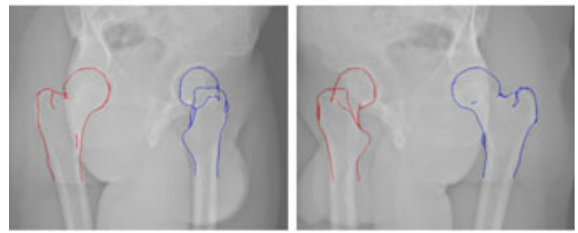

(c)

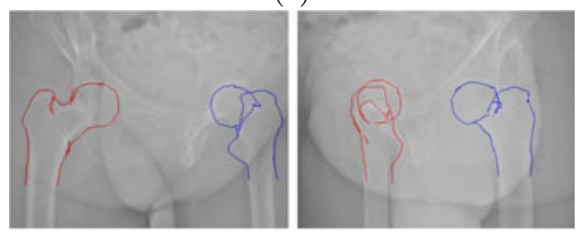

(d)

Fig. 2. Experimental Results. (a) Four 3D surface reconstruction results with pointto-surface errors on femoral head. (b) Boxplots on the DICE, the mean and STD of the point-to-surface errors $(\mathrm{mm})$. On each box, the central mark in red is the median, the edges of the box are the 25th and 75th percentiles, the whiskers extend to the most extreme data points. (c) and (d) Results on in vivo data, where projected boundaries of the estimated femurs in the bi-planar views are shown for each example. 


\section{Conclusion}

In this paper, we have proposed a novel approach to 3D femur estimation from biplanar X-ray views. The main innovations consist in the multi-resolution shape representation, the ability to model pose/mirror-symmetry invariant prior and the image likelihood through local interactions, and the higher-order MRF formulation of the surface estimation which is solved by dual-decomposition optimization. Promising results demonstrate the potential of the method.

Future work consists of introducing a joint model that couples femur with the hipbone socket which could enhance the diagnostic potential of the method, and combining distinctive (visual) anatomical landmarks with the existing formulation towards increasing precision and the overall performance. Last, the application of the method to other clinical settings also bears great promises.

\section{References}

1. Cootes, T.F., Taylor, C.J., Cooper, D.H., Graham, J.: Active shape models - their training and application. CVIU 61(1), 38-59 (1995)

2. Glocker, B., Komodakis, N., Tziritas, G., Navab, N., Paragios, N.: Dense image registration through mrfs and efficient linear programming. Medical Image Analysis 12(6), 731-741 (2008)

3. Gottschling, H., Roth, M., Schweikard, A., Burgkart, R.: Intraoperative, fluoroscopy-based planning for complex osteotomies of the proximal femur. Int. J. Med. Robotics Comput. Assist. Surg. 1, 67-73 (2005)

4. Heimann, T., Meinzer, H.P.: Statistical shape models for 3d medical image segmentation: A review. Medical Image Analysis 13(4), 543-563 (2009)

5. Kadoury, S., Cheriet, F., Laporte, C., Labelle, H.: A versatile 3d reconstruction system of the spine and pelvis for clinical assessment of spinal deformities. Med. Biol. Engineering and Computing 45(6), 591-602 (2007)

6. Komodakis, N., Paragios, N., Tziritas, G.: MRF optimization via dual decomposition: Message-passing revisited. In: ICCV (2007)

7. Komodakis, N., Paragios, N., Tziritas, G.: Clustering via lp-based stabilities. In: NIPS (2009)

8. Kurazume, R., Nakamura, K., Okada, T., Sato, Y., Sugano, N., Koyama, T., Iwashita, Y., Hasegawa, T.: 3d reconstruction of a femoral shape using a parametric model and two 2d fluoroscopic images. CVIU 113(2), 202-211 (2009)

9. Laporte, S., Skalli, W., de Guise, J.A., Lavaste, F., Mitton, D.: A biplanar reconstruction method based on $2 \mathrm{~d}$ and $3 \mathrm{~d}$ contours: Application to the distal femur. Computer Methods in Biomechanics and Biomedical Engineering 6(1), 1-6 (2003)

10. Mitton, D., Deschenes, S., Laporte, S., Godbout, B., Bertrand, S., de Guise, J.A., Skalli, W.: 3d reconstruction of the pelvis from bi-planar radiography. Computer Methods in Biomechanics and Biomedical Engineering 9(1), 1-5 (2006)

11. Varshney, K.R., Paragios, N., Deux, J.F., Kulski, A., Raymond, R., Hernigou, P., Rahmouni, A.: Postarthroplasty examination using x-ray images. IEEE Trans. Med. Imaging 28(3), 469-474 (2009)

12. Wang, C., Teboul, O., Michel, F., Essafi, S., Paragios, N.: 3d knowledge-based segmentation using pose-invariant higher-order graphs. In: Jiang, T., Navab, N., Pluim, J.P., Viergever, M.A. (eds.) MICCAI 2010, Part III. LNCS, vol. 6363, pp. 189-196. Springer, Heidelberg (2010) 